\title{
Proprioceptive neuromuscular facilitation training induced alterations in muscle fibre type and cross sectional area
}

\author{
N Kofotolis, I S Vrabas, E Vamvakoudis, A Papanikolaou, K Mandroukas
}

Br J Sports Med 2005;39:el 1 (http://www.bisportmed.com/cgi/content/full/39/3/el 1). doi: 10.1136/bjsm.2004.010124

See end of article for authors' affiliations

(1)

Correspondence to: Dr Mandroukas Department of Physical

Education and Sports

Science, Aristotle

University of Thessaloniki,

University Campus,

Thessaloniki 54124

Greece; kmandrou@

phed.auth.gr

Accepted 6 April 2004
Objectives: To compare the effects of proprioceptive neuromuscular facilitation (PNF) and isokinetic training on fibre type distribution and cross sectional area of the vastus lateralis muscle.

Methods: Twenty four male university students were divided into two equal groups: PNF training and isokinetic training (ISO). The training regimen for the PNF group consisted of three sets of 30 repetitions against maximal resistance, alternating two patterns of sequential movements of the right lower extremity: (a) toe flexion and ankle plantar flexion and eversion; (b) knee extension and hip extension, abduction, and internal rotation. The ISO group performed three sets of 30 repetitions alternating knee extension and flexion of the right leg at angular velocities of 180 and $90 \%$ in an isokinetic dynamometer (Cybex). Both groups trained three times a week for a total of eight weeks. Muscle biopsy specimens were obtained from the right vastus lateralis muscle before and after training.

Results: The mean percentage area of type IIB fibre was significantly decreased $(p<0.01)$ after eight weeks of PNF training, whereas that of type IIA fibre was significantly $(p<0.05)$ increased. The mean percentage area of ISO trained type IIAB fibres exhibited an augmentative pattern $(p<0.01)$ with a parallel reduction $(p<0.05)$ in type IIA. Percentage fibre type distribution exhibited a similar pattern. Conclusions: Both PNF and ISO training alter fibre type distribution and mean cross sectional area. These changes occur in the type II fibre subgroup.
$\mathrm{P}$ roprioceptive neuromuscular facilitation (PNF) exercises are designed to promote the neuromuscular response of the proprioceptors. PNF patterns have a spiral, diagonal direction and are in line with the topographical arrangement of the muscles. facilitating the activation of biarticular muscles. ${ }^{1}$ This means that PNF exercises are very similar to the actions and movements found in various sports. They therefore appear to be more suitable for performance enhancement than conventional weight training programmes. PNF is therefore often used as an alternative form of progressive resistive exercise by physiotherapists as its use should be more advantageous than the usual strength programmes in athletic injury rehabilitation. ${ }^{2}$ Nelson et $a l^{3}$ compared the effects of PNF and weight training. They reported similar strength gains, but PNF was better at enhancing performance, as evidenced by the larger gains in throwing distance and jumping height.

The use of isokinetic exercise in rehabilitation, conditioning, and research has become widespread since the development of modern isokinetic dynamometers, which provide data on the mechanical properties of muscle under conditions of constant velocity in vivo.

Cross sectional studies, using histochemical methods on skeletal muscle from various groups of elite athletes, suggest that the pattern of physical activity affects muscle fibre type predominance. ${ }^{45}$ More recent longitudinal studies have shown changes in the histochemical fibre type profile after a period of increased physical activity. ${ }^{67}$

The effects of PNF training on skeletal muscle fibre composition are not known. Based on the evidence that the pattern of physical activity affects muscle fibre type predominance, we hypothesised that PNF, like isokinetic training, would affect vastus lateralis muscle fibre type predominance and mean area, and that these changes would appear in the type II fibre subgroup.

\section{METHODS \\ Subjects}

Twenty four male university students were informed of the test procedure, purpose, and known risks before giving their informed consent to participate. The subjects were divided into two equal groups: (a) PNF training (mean (SEM) age 22.2 (1.3) years, height $178.2(5.3) \mathrm{cm}$, weight $74.5(9.2) \mathrm{kg})$; (b) isokinetic training (ISO; age 20.5 (1.7) years, height 178.9 (6.7) $\mathrm{cm}$, weight $73.3(9.5) \mathrm{kg}$ ).

\section{PNF training}

The training regimen for the PNF group consisted of three sets of 30 repetitions against maximal resistance, alternating two patterns of sequential movements of the right lower extremity: (a) flexion-adduction-external rotation with knee extension; $(b)$ extension-abduction-internal rotation with knee flexion. The first pattern consisted of the following movement components: toes flex and adduct (lateral toes more than medial) toward fibular side; foot and ankle perform plantar flexion with eversion; knee flexes with tibia internally rotating on femur; hip extends, abducts, and internally rotates. The second pattern consisted of the following movement components: toes extend and abduct (medial toes more than lateral) toward tibial side; foot and ankle dorsiflex with inversion; knee extends with tibia externally rotating on femur; hip flexes, adducts, and externally rotates. Rest intervals of 30 and 60 seconds were allowed after the completion of 10 repetitions for each pattern and between sets respectively.

\section{Isokinetic training}

The ISO group followed a similar pattern of training, performing three sets of 30 repetitions alternating knee

Abbreviations: $\mathrm{MHC}$, myosin heavy chain; PNF, proprioceptive neuromuscular facilitation 
Table 1 Percentage muscle fibre area before and after training

\begin{tabular}{lllll}
\hline Training & Fibre type & Before & After & p Value \\
\hline PNF & I & $44.49(8.9)$ & $40.12(8.5)$ & NS \\
& IIA & $39.99(8.7)$ & $46.34(9.5)$ & 0.05 \\
& IIAB & $5.67(4.0)$ & $8.25(7.4)$ & NS \\
& IIB & $8.85(7.9)$ & $0.58(1.9)$ & 0.01 \\
Isokinetic & IIC & $2.36(4.0)$ & $5.11(7.2)$ & NS \\
& I & $37.26(10.8)$ & $37.46(9.3)$ & NS \\
& IIA & $46.61(9.1)$ & $37.06(12.6)$ & 0.05 \\
& IIAB & $5.10(6.7)$ & $17.24(9.1)$ & 0.004 \\
& IIB & $10.28(9.5)$ & $6.56(8.4)$ & NS \\
& IIC & $1.83(1.5)$ & $1.93(3.2)$ & NS
\end{tabular}

Values are mean (SD).

PNF, Proprioceptive neuromuscular facilitation.

extension and flexion of the right leg at angular velocities of 180 and $90 \%$ in a speed controlled isokinetic dynamometer (Cybex 6000; Lumex Inc, Ronkonkoma, New York, USA). The same rest intervals as described above were allowed. Both groups trained three times a week for a total of eight weeks.

\section{Muscle biopsies}

Needle biopsy specimens (100-150 mg) were taken by suction from the middle portion of the vastus lateralis from the subjects' right leg. ${ }^{8}$ The muscle specimens were trimmed, mounted, and frozen in isopentane, which was cooled with nitrogen at $-80^{\circ} \mathrm{C}$, and analysed histochemically.

\section{Histochemistry}

Serial transverse sections $(10 \mu \mathrm{m})$ were cut in a cryotome at $-20^{\circ} \mathrm{C}$. The sections were mounted on coverslips and stained for myofibrillar ATPase after preincubation at $\mathrm{pH} 4.3,4.6$, and 10.4 for the classification of fibre type distribution. Fibres that were stable at $\mathrm{pH} 10.4$ and 4.6 but labile at $\mathrm{pH} 4.3$ were classified as type IIB (fast twitch glycolytic fibres) or type IIAB, depending on their staining intensities at pH 4.6 (type IIB are stained more darkly than type IIAB). About 400 fibres were classified in each sample. Fibre areas from the ATPase stained sections were analysed. Fibres were counted and areas measured using a computer based image analysis system including TEMA video analysis software (Scan Beam, Hadsund, Denmark) integrated with a high resolution colour video camera (JVC, Yokosuka, Japan).

\section{Statistical analysis}

A one way analysis of variance was used to determine differences in all variables after training. Significance was established at $\mathrm{p}<0.05$. Data are expressed as mean (SD).

\begin{tabular}{lllll}
$\begin{array}{c}\text { Table } 2 \\
\text { training }\end{array}$ & \multicolumn{4}{l}{ Fibre type distribution (\%) before and after } \\
\hline Training & Fibre type & Before & After & p Value \\
\hline PNF & I & $47.50(10.5)$ & $43.88(10.1)$ & NS \\
& IIA & $36.18(8.8)$ & $42.31(10.8)$ & NS \\
& IIAB & $5.81(4.2)$ & $8.97(7.6)$ & NS \\
& IIB & $9.68(9.6)$ & $0.67(2.2)$ & 0.016 \\
& IIC & $2.12(3.5)$ & $4.62(6.3)$ & NS \\
Isokinetic & I & $40.03(10.3)$ & $39.69(10.2)$ & NS \\
& IIA & $42.43(9.9)$ & $32.87(11.5)$ & 0.040 \\
& IIAB & $5.40(7.0)$ & $17.92(8.7)$ & 0.003 \\
& IIB & $10.76(9.4)$ & $7.85(10.3)$ & NS \\
& IIC & $1.87(1.5)$ & $1.99(3.0)$ & NS \\
\hline
\end{tabular}

Values are mean (SD).

PNF, Proprioceptive neuromuscular facilitation.
Table 3 Cross sectional area $\left(\mu \mathrm{m}^{2}\right)$ of fibres before and after training

\begin{tabular}{lllll}
\hline Training & Fibre type & Before & After & p Value \\
\hline PNF & I & $4728.0(1298)$ & $4829.0(1038)$ & NS \\
& IIA & $5503.0(1110)$ & $5828.0(1251)$ & NS \\
& IIAB & $3541.0(2364)$ & $2951.7(2582)$ & NS \\
& IIB & $3406.8(2287)$ & $1106.5(2492)$ & 0.01 \\
& IIC & $2368.4(3535)$ & $3408.4(3384)$ & NS \\
Isokinetic & I & $4204.4(727)$ & $4670.1(1014)$ & NS \\
& IIA & $5118.6(937)$ & $5647.2(1451)$ & NS \\
& IIAB & $2052.5(2223)$ & $4695.4(1594)$ & 0.01 \\
& IIB & $3373.4(2286)$ & $2389.7(2709)$ & NS \\
& IIC & $3368.5(2212)$ & $2754.5(2617)$ & NS \\
\hline
\end{tabular}

Values are mean (SD).

PNF, Proprioceptive neuromuscular facilitation.

\section{RESULTS}

The mean percentage area of type IIB fibre was significantly $(p<0.01)$ decreased after eight weeks of PNF training, whereas that of type IIA fibre was significantly $(\mathrm{p}<0.05)$ increased (table 1). The mean percentage area of ISO trained type IIAB fibre exhibited an augmentative pattern $(\mathrm{p}<0.01)$ with a parallel reduction $(\mathrm{p}<0.05)$ in type IIA.

Percentage fibre type distribution exhibited a similar pattern, with a significant $(\mathrm{p}<0.05)$ decrease in type IIB after eight weeks of PNF training. Percentage fibre type distribution after ISO training exhibited an augmentative pattern $(\mathrm{p}<0.01)$ in type IIAB with a parallel significant $(p<0.05)$ reduction in type IIA (table 2$)$.

The cross sectional area of type IIB fibres in the PNF trained group had significantly $(\mathrm{p}<0.01)$ decreased, and that of type IIAB fibres in the ISO trained group had significantly $(\mathrm{p}<0.01)$ increased (table 3$)$.

\section{DISCUSSION}

\section{Overview of principle findings}

This study compared the effects of PNF and isokinetic training on the fibre type distribution and cross sectional area of vastus lateralis muscle. We hypothesised that PNF, like isokinetic training, would affect muscle fibre type predominance and mean area, and that these changes would appear in the type II fibre subgroup. The mean percentage area of type IIB fibre was significantly decreased after eight weeks of PNF training with a concomitant increase in type IIA. Percentage fibre type distribution exhibited a similar pattern. Isokinetic training induced a significant increase in type IIAB fibre type distribution with a concomitant decrease in type IIA. Hence these data clearly indicate that PNF training alters fibre type distribution and mean area and that these changes occur in the type II fibre subgroup and follow a unidirectional pattern of transformation (fast to slow), partially supporting our initial hypothesis. In contrast, isokinetic training induced alterations seem to follow the opposite directional pattern from type IIA towards type IIAB. A detailed discussion of these and related issues follows.

\section{Fibre type distribution and mean area}

Studies of transformation of fibre types have suggested that the response of human skeletal muscle to intensive endurance training is a transformation from histochemical type II fibres to type I fibres. ${ }^{90}$ Other studies have also suggested that strength training induces a histochemical fibre type change from type IIB to type IIA. ${ }^{6711} 12$ These transformations which occur with different kinds of training or detraining inflicted on the human skeletal muscle therefore seem to follow a unidirectional pattern from fast to slow $\left(\mathrm{IIB} \rightarrow \mathrm{IIA} \rightarrow \mathrm{I}\right.$ ) or vice versa. ${ }^{10}{ }^{13}{ }^{14}$ Our findings support the unidirectional pattern of transformation occurring in the type 
What is known on this topic

The pattern of physical activity affects muscle fibre type predominance. PNF exercises are designed to promote the neuromuscular response of the proprioceptors, but the effects of PNF training on skeletal muscle fibre composition are not known. The use of isokinetic exercise in rehabilitation, conditioning, and research is widespread.

II fibre subgroup, as eight weeks of PNF training caused a significant decrease in type IIB fibre type distribution and eight weeks of isokinetic training caused a reduction in type IIA. The differential response of the muscle to the two different types of stimulus imposed in this study is not well understood.

One possible explanation may lay in the fact that the biopsy specimens in our study were taken from the vastus lateralis muscle, on which the two different kinds of training probably imposed different loads. It is well known that PNF patterns have a spiral, diagonal direction in line with the topographical arrangement of the muscles and are very similar to the actions and movements found in various sports, facilitating the activation of biarticular muscles. The subjects in our PNF group were trained against maximal resistance, alternating two patterns of sequential movements of the right lower extremity: (a) toe flexion and ankle plantar flexion and eversion; $(b)$ knee extension and hip extension, abduction, and internal rotation. It is logical therefore to reason that these movements recruited a lot of different synergistic muscles and imposed a different load on the quadriceps and hamstring muscles from that imposed by knee extension and flexion respectively, performed in a speed controlled isokinetic dynamometer, with stabilisation straps at the trunk, thigh, and tibia to prevent extraneous joint movement. The higher intensity imposed on the vastus lateralis muscle during isokinetic training compared with PNF training may therefore explain the opposite directional pattern of transformation from type IIA towards type IIAB fibres evidenced by the histochemical analysis.

Another possible explanation that cannot be excluded is that the isokinetic training stimuli administered in this case had a combination of resistance and endurance characteristics, imposed under constant speed throughout the range of motion. In contrast, during PNF training, the resistance to the limb was imposed by the physiotherapist without any control of the speed. Similarly to our findings, increased expression of myosin heavy chain (MHC) isoform IIA due to sprint training, which was related to bidirectional transformation from both MHC isoforms I and IIB towards MHC isoform IIA, was reported by Andersen et al. ${ }^{15}$ These authors analysed the MHC composition of single fibres from the vastus lateralis muscle of a group of male sprinters, before and after a three month period of intensive strength and interval training. Our findings are only partially in line with the above as isokinetic training induced a transformation that occurred from type IIA towards type IIAB fibres with no significant reduction of type IIB. Further study is required to resolve this issue.

\section{Changes in cross sectional area}

Several studies have reported hypertrophy in muscle fibres of athletes participating in extremely strength demanding events. ${ }^{16-18}$ Longitudinal studies have also shown enlargement of muscle fibre area after strength training of sedentary subjects. ${ }^{7}$ Our findings that PNF training caused significant decreases in type IIB cross sectional area are in line with these studies.
What this study adds

Both PNF and isokinetic training altered fibre type distribution and mean cross sectional area of the vastus lateralis muscle. These changes occur in the type II fibre subgroup.

Isokinetic training caused significant increases in type IIAB cross sectional area, with a tendency towards reduction in type IIB muscle fibres. As discussed above, the two different kinds of training probably imposed different loads on the muscle examined. Also, the isokinetic training had a combination of resistance and endurance characteristics, imposed under constant speed throughout the whole range of motion.

Data on quadriceps muscle strength of our subjects, not shown here but published previously, ${ }^{19}$ showed that eight weeks of isokinetic training at 180 and $90 \%$, as well as eight weeks of PNF training, produced significant increases in peak torque at speeds slower and faster than the training velocity $(60$ and $300 \%$ s). These findings are in line with those of Caiozzo et al ${ }^{20}$ It is logical therefore to reason that mostly type IIAB fibres were recruited during this type of stimulus and thus the cross sectional area of these fibres was affected, a postulate that is supported by our findings. This contention is also supported by the results of Houston et al, ${ }^{21}$ who reported that 10 weeks of high resistance training increased the cross sectional area of type II fibres by $20 \%$, and the results of Brown et al, 22 who reported that 13 weeks of resistance training increased the cross sectional area of type II fibres by $30 \%$. These findings do not agree with those of Andersen et al, ${ }^{23}$ who reported no significant increase in the muscle fibre area in any of the three histochemically determined fibre types after a three month, high resistance knee extensor strength training programme in soccer players. The reason for the absence of any significant hypertrophy in that study may be that the amount of conducted strength training was too small.

In conclusion, the findings of this study clearly indicate that PNF training alters fibre type distribution and mean area, and that these changes seem to appear in the type II fibre subgroup and follow a unidirectional pattern of transformation (fast to slow). Similarly, isokinetic training induced alterations appear in the type II fibre subgroup, but the opposite pattern is followed, from type IIA to type IIAB, revealing a differential type of loading on the vastus lateralis muscle.

\section{Authors' affiliations}

N Kofotolis, I S Vrabas, E Vamvakoudis, A Papanikolaou,

K Mandroukas, Department of Physical Education and Sports Science, Aristotelian University of Thessaloniki, Thessaloniki, Greece

Competing interests: none declared

\section{REFERENCES}

1 Knott M, Voss DE. Proprioceptive neuromuscular facilitation: patterns and techniques, 2nd ed. New York: Harper and Row Inc, 1968.

2 Lusting A, Ball E, Looney M. A comparison of two proprioceptive neuromuscular facilitation techniques for improving range of motion and muscular strength. Isokinet Exerc Sci 1992;2:154-9.

3 Nelson AG, Chambers RS, McGown CM, et al. Proprioceptive neuromuscular facilitation versus weight training for enhancement of muscular strength and athletic performance. J Sport Phys Ther 1986;7:250-3.

4 Staron RS, Hikida RS, Hagerman FC, et al. Human skeletal muscle fibre type adaptability to various workloads. J Histochem Cytochem 1984;32:146-52.

5 Larsson L, Ansved T. Effects of long term physical training onenzyme histochemical and functional skeletal muscle charachteristics in man. Muscle Nerve 1985;8:714-22.

6 Staron RS, Maclicky ES, Leonardi MJ, et al. Muscle hypertrophy and fast fibre conversions in heavy resistance-trained women. Eur $J$ Appl Physiol 1989;60:21-4. 
7 Hather BM, Tesch PA, Buchanan P, et al. Influence of eccentric actions on skeletal muscle adaptations to resistance training. Acta Physiol Scand 1991; 143:177-85.

8 Bergström J. Muscle electrolytes in man. Scand J Clin Lab Invest 1962;14(suppl 68):1-110.

9 Howald H, Hoppeler H, Claassen $\mathrm{H}$, et al. Influences of endurance training on the ultrastructural composition of different muscle fibre types in humans. Pflugers Arch 1985;403:369-76.

10 Pette D, Vrbova G. Neural control of phenotypic expression in mammalian muscle fibers. Muscle Nerve 1985;8:676-89.

11 Staron RS, Hikida RS, Murray TF, et al. Assessment of skeletal muscle damage in successive biopsies from strength-trained and untrained men and women. Eur J Appl Physiol Occup Physiol 1992;65:258-64.

12 Adams GR, Hather BM, Baldwin KM, et al. Skeletal muscle myosin heavy chain composition and resistance training. J Appl Physiol 1993;74:911-15.

13 Schantz PG. Plasticity of human skeletal muscle. Acta Physiol Scand 1986:128:7-27.

14 Biral D, Betto R, Danieli-Betto D, et al. Myosin heavy chain composition of single fibers from normal human muscle. Biochem $J$ 1988;250:307-8.

15 Andersen JL, Klitgaard H, Saltin B. Myosin heavy chain isoforms in single fibres from $\mathrm{m}$. vastus lateralis of sprinters: influence of training. Acta Physio Scand 1994;151:135-42.
16 Ryushi T, Hakkinen $\mathrm{K}$, Kauhanen $\mathrm{H}$, et al. Muscle fiber characteristics, muscle cross-sectional area and force production in strength athletes, physically active males and females. Scand J Sports Sci 1988;10:7-15.

17 Bell DG, Jacobs I. Muscle fiber area fibre type and capillarization in male and female body builders. Can J Sports Sci 1990;15:115-19.

18 Klitgaard H, Zhou M, Richter EA. Myosin heavy chain composition of single fibers from $\mathrm{m}$. biceps brachii of male body builders. Acta Physiol Scand 1990;140:175-80.

19 Kofotolis N, Vrabas IS, Kalogeropoulou E, et al. Proprioceptive neuromuscular facilitation versus isokinetic training for strength, endurance and jumping performance. Journal of Human Movement Studies and jumping perform

20 Caiozzo VJ, Perrine JJ, Edgerton VR. Training-induced alterations of the in vivo force-velocity relationship of human muscle. J Appl Physiol 1981;51:750-4.

21 Houston ME, Froese EA, Valeriote SP, et al. Muscle performance morphology and metabolic capacity during strength training and detraining: a one leg model. Eur J Appl Physiol 1983;51:25-35.

22 Brown AB, McArtney N, Moroz D, et al. Strength training effects in aging Med Sci Sports Exerc 1988;21(suppl):s164-72.

23 Andersen JL, Klitgaard H, Bangsbo J, et al. Myosin heavy chain isoforms in single fibers from $\mathrm{m}$. vastus lateralis of soccer players: effects of strengthtraining. Acta Physiol Scand 1994;150:21-6. 